\title{
IBP methods at finite temperature
}

\author{
Madoka Nishimura and York Schröder \\ Tohoku University of Community Service and Science, Sakata 998-8580, Japan \\ Faculty of Physics, University of Bielefeld, 33501 Bielefeld, Germany \\ E-mail: madoka.nishimura@koeki-u.ac.jp, yorks@physik.uni-bielefeld.de
}

\begin{abstract}
We demonstrate the applicability of integration-by-parts (IBP) identities in finitetemperature field theory. As a concrete example, we perform 3-loop computations for the thermodynamic pressure of QCD in general covariant gauges, and confirm earlier Feynmangauge results.
\end{abstract}

KeYwords: Thermal Field Theory, QCD, NLO Computations 


\section{Contents}

1 Introduction 1

2 Thermodynamic observables $\quad 2$

3 Integral reduction $\quad 4$

4 Results of diagrammatic calculation $\quad 6$

5 Conclusions $\quad 11$

$\begin{array}{ll}\text { A One- and two-loop vacuum sum-integrals } & 11\end{array}$

B Three-loop vacuum sum-integrals 12

\section{Introduction}

Modern perturbative computations typically involve large numbers of Feynman diagrams and/or Feynman integrals, whose successful evaluation is greatly facilitated by the use of computer algebra. Indeed, in the field of multi-loop calculations (in zero-temperature field theories) the algorithmic formulation is in a quite mature state, as witnessed by numerous higher-order results, such as e.g. 5-loop results for the QED beta function [1], complete 3loop and parts of 4-loop terms in the electron anomalous magnetic moment [2], or 4-loop contributions to electroweak precision observables [3], just to name a few.

Such calculations are typically performed in a sequence of steps: (a) generation of the complete set of diagrams and counter-terms contributing to the observable under study; (b) application of Feynman rules, necessary projectors and traces, performing Lorentz algebra and scalarization; (c) mapping onto a set of integral templates; (d) reduction to a few ("master") integrals; (e) expansion in epsilon.

For each of these distinct steps, a variety of systematic algorithmic methods and tools can be applied, the most commonly used ones being: (a) graph theory (efficiently coded in the package QGRAF [4]); (b,c) computer-algebra systems (such as e.g. FORM [5], Ginac [6], Mathematica [7]); (d) integration-by-parts methods (pioneered by Chetyrkin and Tkachov [8], formalized by Laporta in [9] and implemented e.g. in the public packages Air [10], FIRE [11], Reduze [12]); (e) difference equations [9], differential equations (see, e.g. [13]), harmonic polylogarithms and -sums [14] (where the expansion can be automatized for simple 0-scale problems [15], but is non-trivial for multiple-scale problems), graph polynomials [16], sector decomposition [17]. 
For problems in finite temperature field theories, however, a similar computer-algebraic approach to higher-order calculations is much less developed, although a limited number of results up to the three-loop [18-20] and even the four-loop level [21] do exist. Whenever gauge theories are treated, owing to the structure of gauge-field propagators and -vertices, most authors choose to work in a fixed (typically Feynman) gauge, in order to reduce the complexity of the calculation.

While most of these works were performed in a more traditional way, there is no major obstacle in carrying over the systematics of the modern computer-algebraic developments mentioned above to finite temperature calculations, which as a consequence abolishes the need to work in fixed gauges. In fact, for the steps (a) to (d) - i.e. diagram generation / algebraic simplifications / mapping / reduction - the methods from zero-temperature field theory can be generalized directly, with a few minor modifications. It is only step (e) - i.e. the epsilon expansion of master integrals - that resists full automation, owing to the fact that the available techniques for sum-integrals which are needed in the thermal setting are much more limited than those for pure continuum integrals (for which at least numerical methods are guaranteed to work). For first attempts in treating whole classes (as opposed to treating them one-by-one as has been the state of the art previously [18, 21, 22]) of non-trivial sum-integrals, see [23].

In this paper, we want to demonstrate the utility of computer-algebra methods for thermal field theories, which mainly concerns the integral reduction step (d). Concentrating on the concrete example of the 3-loop free energy of hot QCD, we briefly introduce the corresponding observable in Sec. 2 and then explain the specifics of the (IBP) reduction method generalized to finite temperature in Sec. 3. Sec. 4 contains the result of our diagrammatic calculation, confirming the known 3-loop result [19], however in general covariant gauges. In Sec. 5 we conclude. The Appendix lists some useful sum-integrals that are needed for the final result.

\section{Thermodynamic observables}

QCD equilibrium properties, such as its free energy density $F$, are encoded in the logarithm of the partition function

$$
F=-\frac{T}{V} \ln \int \mathcal{D}\left[A_{\mu}, \bar{\psi}, \psi\right] \exp \left(-\int_{0}^{1 / T} \mathrm{~d} \tau \int \mathrm{d}^{d} x \mathcal{L}_{\mathrm{QCD}}\right) .
$$

Here, $V=\int \mathrm{d}^{d} x$ denotes the spatial volume occupied by the system $(d=3-2 \epsilon)$, and we work in the imaginary time formalism, defined on a $(d+1)$-dimensional Euclidean space with a compact temporal coordinate $\tau$ with period $1 / T$ set by the temperature. Bosonic/fermionic (gluon/quark) fields are periodic/antiperiodic functions of $\tau$. The (Euclidean) QCD Lagrangian reads

$$
\mathcal{L}_{\mathrm{QCD}}=\frac{1}{4} F_{\mu \nu}^{a} F_{\mu \nu}^{a}+\sum_{i=1}^{N_{\mathrm{f}}} \bar{\psi}_{i}\left(\gamma_{\mu} D_{\mu}+m_{i}+i \mu_{i}\right) \psi
$$


with field strength tensor $F_{\mu \nu}^{a}=\partial_{\mu} A_{\nu}^{a}-\partial_{\nu} A_{\mu}^{a}+g f^{a b c} A_{\mu}^{b} A_{\nu}^{c}$ and covariant derivative $D_{\mu}=$ $\partial_{\mu}+i g A_{\mu}^{a} T^{a}$. We will set quark masses $m_{i}$ and chemical potentials $\mu_{i}$ to zero here, and work with the gauge group $\mathrm{SU}\left(N_{\mathrm{c}}\right)$, where $C_{\mathrm{A}}=N_{\mathrm{c}}, \operatorname{Tr}\left(T^{a} T^{b}\right)=\frac{1}{2} \delta^{a b}$ and $T^{a} T^{a}=C_{F} \mathbb{1}=\frac{N_{\mathrm{c}}^{2}-1}{2 N_{\mathrm{c}}} \mathbb{1}$.

It turns out that the weak-coupling expansion of Eq. (2.1) is nonanalytic in the strong coupling constant $\alpha_{s}=g^{2} / 4 \pi$. The physical reason is that, due to multiple interactions in the thermal medium, screening masses are dynamically generated for all massless particles, resulting in a multi-scale system. In fact, at high temperatures, asymptotic freedom guarantees a small gauge coupling $g$. In this regime, QCD develops a hierarchy of three momentum scales $\pi T \gg g T \gg g^{2} T / \pi$, whose effect can be most transparently accounted for in an effective theory setup [24]. Systematically integrating out the largest ("hard") scale $\pi T$ and the second-largest ("soft") scale $g T$ in turn, one obtains a dimensionally reduced effective theory [25] for the smallest ("ultrasoft") scale, which has been dubbed magnetostatic QCD (MQCD) [19]. The leading term of the MQCD action turns out to be a 3-dimensional pure Yang-Mills theory, which is confining and therefore has to be treated with suitable non-perturbative methods [26].

Utilizing the effective theory setup, the QCD pressure can be expressed as [19]

$$
p_{\mathrm{QCD}}(T) \equiv-\lim _{V \rightarrow \infty} F=p_{\text {hard }}(T)+p_{\text {soft }}(T)+p_{\text {ultrasoft }}(T)
$$

where $p_{\text {hard }}$ and $p_{\text {soft }}$ are perturbatively computable matching coefficients which account for contributions from hard and soft momentum scales, respectively. Defining these perturbative matching coefficients in the $\overline{\mathrm{MS}}$ scheme, the remaining contribution

$$
p_{\text {ultrasoft }}(T) \equiv\left\{\lim _{V \rightarrow \infty} \frac{T}{V} \ln \int \mathcal{D}\left[A_{i}\right] \exp \left(-\int \mathrm{d}^{d} x \mathcal{L}_{\mathrm{MQCD}}\right)\right\}^{\overline{\mathrm{MS}}}
$$

entails the effective Lagrangian $\mathcal{L}_{\mathrm{MQCD}}=\frac{1}{4} F_{i j}^{a} F_{i j}^{a}+\ldots$, where $F_{i j}^{a}=\partial_{i} A_{j}^{a}-\partial_{j} A_{i}^{a}+$ $g_{\mathrm{M}} f^{a b c} A_{i}^{b} A_{j}^{c}$ contains the 3 -dimensional gauge coupling $g_{\mathrm{M}}$. In contrast to the dimensionless 4dimensional gauge coupling $g$, the dimensionality of $g_{\mathrm{M}}^{2}$ is $\mathrm{GeV}$, such that for dimensional reasons $p_{\text {ultrasoft }} \sim T g_{\mathrm{M}}^{6}$. On the other hand, perturbative matching yields $g_{\mathrm{M}}^{2}=g^{2} T(1+\mathcal{O}(g))$,

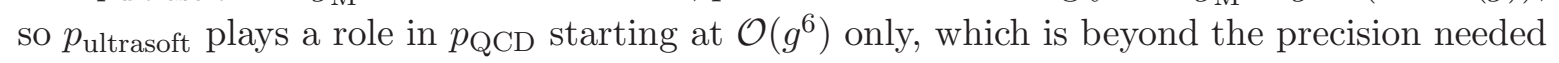
for our investigation (see, however [26]).

The two matching coefficients in Eq. (2.3) are defined in analogy to Eq. (2.4), but with actions $\int_{0}^{1 / T} \mathrm{~d} \tau \int \mathrm{d}^{d} x \mathcal{L}_{\mathrm{QCD}}$ and $\int \mathrm{d}^{d} x \mathcal{L}_{\mathrm{EQCD}}$ for $p_{\text {hard }}$ and $p_{\text {soft }}$, respectively. The latter depends on the so-called 3d electrostatic QCD (EQCD) Lagrangian containing a massless gauge field $A_{i}$ and a massive adjoint scalar $A_{0}$, and whose structure results from integrating out the hard scales from QCD, yielding the leading terms $\mathcal{L}_{\mathrm{EQCD}}=\frac{1}{4} F_{i j}^{a} F_{i j}^{a}+\operatorname{Tr}\left[D_{i} A_{0}\right]\left[D_{i} A_{0}\right]+m_{\mathrm{E}}^{2} \operatorname{Tr} A_{0}^{2}+$ $\lambda_{\mathrm{E}}^{(1)}\left(\operatorname{Tr} A_{0}^{2}\right)^{2}+\lambda_{\mathrm{E}}^{(2)} \operatorname{Tr} A_{0}^{4}+\ldots$ For a review on the status of the different contributions we refer to [27].

It turns out that at present, the bottleneck is the evaluation of higher orders in $p_{\text {hard }}(T)$, which can be obtained by adding all vacuum diagrams in 4-dimensional thermal QCD, evaluated in the "naive" perturbative sense, i.e. by regularizing ultraviolet as well as infrared 
divergences in dimensionally (it is the effective theory setup Eq. (2.3) that properly accounts for infrared effects [28] that need to be resummed). The need to ultimately perform 4-loop computations within this setting is our main motivation to proceed with computer-algebraic methods as far as possible. As mentioned in the introduction, this concerns mainly the task of integral reduction, which we will now discuss, and then apply to the problem of 3-loop corrections to $p_{\text {hard }}(T)$, enabling a comparison with known results from the literature. Going a step beyond the Feynman-gauge treatment of [19], we work in covariant gauges with gluon propagator

$$
D_{\mu \nu}^{a b}(P)=\delta^{a b}\left[\frac{\delta_{\mu \nu}}{P^{2}}-\xi \frac{P_{\mu} P_{\nu}}{\left(P^{2}\right)^{2}}\right]
$$

explicitly keeping the gauge parameter $\xi$ (note that in our convention, $\xi=0(1)$ corresponds to Feynman (Landau) gauge, respectively), and demonstrating its cancellation in the sum of diagrams.

\section{Integral reduction}

For the sake of concreteness, and to avoid too generic notation and proliferation of indices, let us take the problem of three-loop sum-integral reduction as an example here. While it should be understood that the methods introduced below are independent of this choice, let us note that this is in fact the first non-trivial loop order in the case of sum-integrals (cf. Appendix A), and also precisely the level of the computation displayed in Sec. 4.

In general, 3-loop vacuum-type sum-integrals can be written in the form

$$
I_{a b c d e f ; c_{p} c_{q} c_{r}}^{\alpha \beta \gamma} \equiv \sum_{P Q R} \frac{\left(P_{0}\right)^{\alpha}\left(Q_{0}\right)^{\beta}\left(R_{0}\right)^{\gamma}}{\left[P^{2}\right]^{a}\left[Q^{2}\right]^{b}\left[R^{2}\right]^{c}\left[(P-Q)^{2}\right]^{d}\left[(P-R)^{2}\right]^{e}\left[(Q-R)^{2}\right]^{f}},
$$

where $P^{2}=\left(P_{0}\right)^{2}+\mathbf{p}^{2}=\left(\left[2 n_{p}+c_{p}\right] \pi T\right)^{2}+\mathbf{p}^{2}$ are bosonic (fermionic) loop momenta for $c_{i}=0$ (1), and where the indices $a \ldots f \in \mathbb{Z}$ and $\alpha \ldots \gamma \in \mathbb{N}_{0}$. The sum-integral symbol in Eq. (3.1) is a shorthand for

$$
\sum_{P} \equiv \mu^{2 \epsilon} T \sum_{P_{0}} \int \frac{\mathrm{d}^{d} \mathbf{p}}{(2 \pi)^{d}}
$$

where $\mu$ is the minimal subtraction (MS) scheme scale parameter, we take $d=3-2 \epsilon$, and the sum is over all integers $n_{p} \in \mathbb{Z}$. Hence, the set of indices of the sum-integral $I$ enumerates all possible structures that can occur in a particular (3-loop) computation of finite-temperature Feynman integrals without further external momentum scales (a generalization to $n$-point functions or to internal masses and/or chemical potentials is straightforward, but let us focus on the problem at hand here).

Generic integration-by-parts (IBP) relations then provide linear relations between the set of sum-integrals $I$ of Eq. (3.1), using that the integral of a total derivative (here with respect 
to the spatial loop momenta only) vanishes in dimensional regularization,

$$
0=\sum_{P \ldots} \partial_{p_{i}} f_{i}\left(p_{i}, P_{0}, \ldots\right) g(P, \ldots)
$$

with arbitrary function $f_{i}$ (and where $g$ denotes an integrand of the type Eq. (3.1)). The linear relations are obtained by choosing the function $f_{i}$, working out the derivatives, and reexpressing the result in terms of the generic form Eq. (3.1). From this point on, it is clear that the well-established (zero-temperature) algorithms that systematically solve such systems of linear IBP relations can be taken over. In practice, providing a unique ordering relation among the sum-integrals $I$, we use two Laporta-type [9] algorithms (one programmed in FORM [5], as well as one in Ruby [29]; the latter code is used as a cross-check on the former, setting the dimension $d$ to a numerical value, utilizing the speed of numerical Gaussian elimination, and avoiding polynomial algebra).

Furthermore, to reduce the number of relations, it is useful to exploit symmetries among the $I$, which can be generated by linear shifts of loop-momenta. For example, due to the symmetry of the generic sum-integral in Eq. (3.1) it is sufficient to consider, from all $2^{3}=8$ possibilities, the three cases $\left(c_{p}, c_{q}, c_{r}\right)=\{(000),(100),(110)\}$. This mapping can be automated by systematically treating linear relations originating from momentum shifts on the same footing as the IBP relations. A typical relation originating from such shifts is $I_{000111 ; 001}^{000}=I_{111000 ; 110}^{000}$. In general, however, for non-zero coefficients $\alpha, \beta, \gamma$ and/or negative values among the indices $a-f$ (i.e. scalar products in the numerator), these relations have more than one term on the right-hand side.

One could suspect that there exist additional IBP-type relations that take into account the structure of the Matsubara sums, which Eq. (3.3) has not sampled yet. This is not the case, however, as we will show here. In fact, acting with the operator $T \partial_{T}$ on a generic $L$-loop sum-integral (assuming massless propagators, which however can be of bosonic or fermionic type), one can either use the fact that, due to the absence of any other dimensional scale, the dimension of the sum-integral is carried by the scale $T$ only, or apply the derivative to the explicit (in front of each sum) and implicit (in the $P_{0}=(2 n+c) \pi T$ of the integrand) occurrences of $T$ directly

$$
\begin{aligned}
0 & =\left\{T \partial_{T}-T \partial_{T}\right\} T \sum_{P_{10}} \ldots T \sum_{P_{L 0}} \int_{p_{1}} \ldots \int_{p_{L}} g\left(P_{1}, \ldots, P_{L}\right) \\
& =\sum_{P_{1} \ldots P_{L}}\left\{\operatorname{Dim}_{\text {Int }}-L-\sum_{i=1}^{L} P_{i 0} \partial_{P_{i 0}}\right\} g\left(P_{1}, \ldots, P_{L}\right) .
\end{aligned}
$$

It turns out that Eq. (3.4) carries the same information as the sum of the "diagonal" IBP relations

$$
0=\sum_{P_{1} \ldots P_{L}}\left\{\partial_{\mathbf{p}_{1}} \cdot \mathbf{p}_{1}+\partial_{\mathbf{p}_{2}} \cdot \mathbf{p}_{2}+\cdots+\partial_{\mathbf{p}_{L}} \cdot \mathbf{p}_{L}\right\} g\left(P_{1}, \ldots, P_{L}\right)
$$


and therefore does not need to be considered. We have checked this explicitly up to four loops, for vacuum sum-integrals.

One can however use an additional set of relations that derive from the sum-part of the sum-integrals. These additional relations essentially mix bosonic with fermionic sumintegrals, and are based on scaling arguments, such as used e.g. in Appendix B of [30] (see also [31]). One first re-scales the spatial integration momenta of a given bosonic integral as $p_{i} \rightarrow 2 p_{i}$ and then partitions the Matsubara sums as

$$
\sum_{n \in \mathbb{Z}}=\sum_{\text {even }}+\sum_{\text {odd }}
$$

In practice, this provides a few linear relations among different bosonic and fermionic master sum-integrals, which remain after systematic use of the IBP relations Eq. (3.3). The simplest example is the relation between 1-loop tadpoles, as shown in Eq. (A.2). For the 3-loop calculation presented in Sec. 4 below, we obtain (letting $I_{110011 ; a b c}^{000} \equiv I_{a b c}$ for brevity)

$$
\begin{aligned}
I_{000} & =\sum_{P Q R} \frac{1}{P^{2} Q^{2}(P-R)^{2}(Q-R)^{2}} \\
& =T^{3} \sum_{n_{p} \in \mathbb{Z}} \sum_{n_{q} \in \mathbb{Z}} \sum_{n_{r} \in \mathbb{Z}} \int_{\mathbf{p q r}} \frac{1}{\left[\left(2 n_{p} \pi T\right)^{2}+\mathbf{p}^{2}\right]\left[\left(2 n_{q} \pi T\right)^{2}+\mathbf{q}^{2}\right] \ldots} \\
& =2^{3 d-8} T^{3} \sum_{n_{p} \in \mathbb{Z}} \sum_{n_{q} \in \mathbb{Z}} \sum_{n_{r} \in \mathbb{Z}} \int_{\mathbf{p q r}} \frac{1}{\left[\left(n_{p} \pi T\right)^{2}+\mathbf{p}^{2}\right]\left[\left(n_{q} \pi T\right)^{2}+\mathbf{q}^{2}\right] \ldots} \\
& =2^{3 d-8}\left(I_{000}+I_{001}+I_{010}+I_{100}+I_{110}+I_{101}+I_{011}+I_{111}\right) \\
& =2^{3 d-8}\left(I_{000}+6 I_{001}+I_{110}\right),
\end{aligned}
$$

where in the third line we have scaled the spatial momenta, in the fourth line considered all cases of Eq. (3.6) (cubed), and finally exploited symmetries of this simple basketball-type sum-integral. Altogether, we therefore have the linear relation (anticipating the notation of Eqs. (4.11)ff for master integrals)

$$
0=\left(1-2^{8-3 d}\right) I_{000}+6 I_{001}+I_{110}=\left(1-2^{8-3 d}\right) B_{2}+6 F_{6}+F_{7}
$$

between one bosonic and two fermionic integrals, which will allow us to reduce the basis of master integrals by one. We feed these types of linear relations into our IBP system as well.

\section{Results of diagrammatic calculation}

Let us now evaluate the coefficient $p_{\text {hard }}(T)$, as defined in Sec. 1, to three-loop order. In doing so, we work in covariant gauges (cf Eq. (2.5)), aiming at proving gauge parameter independence as well as confirming the corresponding Feynman-gauge result of [19]. Working in dimensional regularization with $d=3-2 \epsilon$, let us rewrite bare quantities as

$$
p_{\mathrm{h}}(T) \equiv \mu^{2 \epsilon} p_{\text {hard }}^{\text {bare }}(T), \quad g^{2} \equiv \mu^{-2 \epsilon} g_{\text {bare }}^{2} .
$$




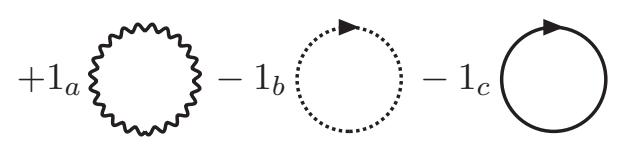

Figure 1. "One-loop" contributions to the QCD pressure. Wiggly/dotted/solid lines denote gluons/ghosts/quarks, respectively. The alphabetical index on the prefactor labels the diagram.

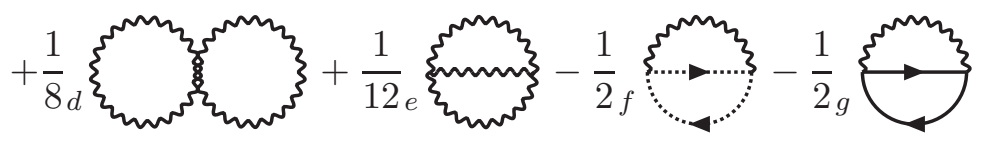

Figure 2. Two-loop contributions to the pressure. Notation as in Fig. (1)

The leading ("one-loop" - or, more precisely, the logarithms of Gaussian path integrals over the quadratic parts of the action, given by the sum over logarithms of momentum-space eigenvalues of the corresponding matrix kernels) terms of Fig. (1) can be expressed in terms of basic logarithmic integrals, which in turn can be related to the more conventional one-loop sum-integrals over propagator structures $I_{m}^{n}$ given in Eq. (A.1). For the bosonic case,

$$
I \equiv \oint_{P} \ln \left(P^{2}\right) \quad \Rightarrow \quad T^{2} \partial_{T^{2}} I=\frac{d+1}{2} I=\frac{1}{2} I+\sum_{P} \frac{P_{0}^{2}}{P^{2}} \quad \Leftrightarrow \quad I=\frac{2}{d} \oint_{P} \frac{P_{0}^{2}}{P^{2}}=\frac{2}{d} I_{1}^{2} .
$$

Here, we noticed that $I$ depends on the scale $T$ only, such that on the one hand its derivative gives the overall dimension, while on the other hand the derivative can be applied directly (hitting the explicit $T$ in the sum-integral as well as the implicit one in $P^{2}$ ). In complete analogy, one gets for the fermionic case

$$
\hat{I} \equiv \sum_{P_{f}} \ln \left(P^{2}\right)=\frac{2}{d} \hat{I}_{1}^{2}=\frac{2}{d}\left(2^{-d}-1\right) I_{1}^{2}
$$

such that the individual diagrams of Fig. (1) contribute as

$$
\begin{array}{ll}
p_{\mathrm{h}}^{[a]}=-\frac{d+1}{d} d_{\mathrm{A}} I_{1}^{2} & \stackrel{d=3}{=} T^{4} \frac{2 \pi^{2}}{45} d_{\mathrm{A}}, \\
p_{\mathrm{h}}^{[b]}=\frac{2}{d} d_{\mathrm{A}} I_{1}^{2} & \stackrel{d=3}{=}-T^{4} \frac{\pi^{2}}{45} d_{\mathrm{A}}, \\
p_{\mathrm{h}}^{[c]}=\frac{4}{d}\left(2^{-d}-1\right) N_{\mathrm{f}} C_{\mathrm{A}} I_{1}^{2} \stackrel{d=3}{=} T^{4} \frac{7 \pi^{2}}{180} N_{\mathrm{f}} C_{\mathrm{A}},
\end{array}
$$

whose sum gives the well-known (QCD version of the) Stefan-Boltzmann law. One can see clearly the effect of the ghosts here, which cancel half of the result of the pure gluonic contribution.

After reduction of the 2-loop diagrams of Fig. (2), we obtain $d$-dimensional bare results, expressed in terms of bosonic as well as fermionic 1-loop tadpoles (as has been pointed out 


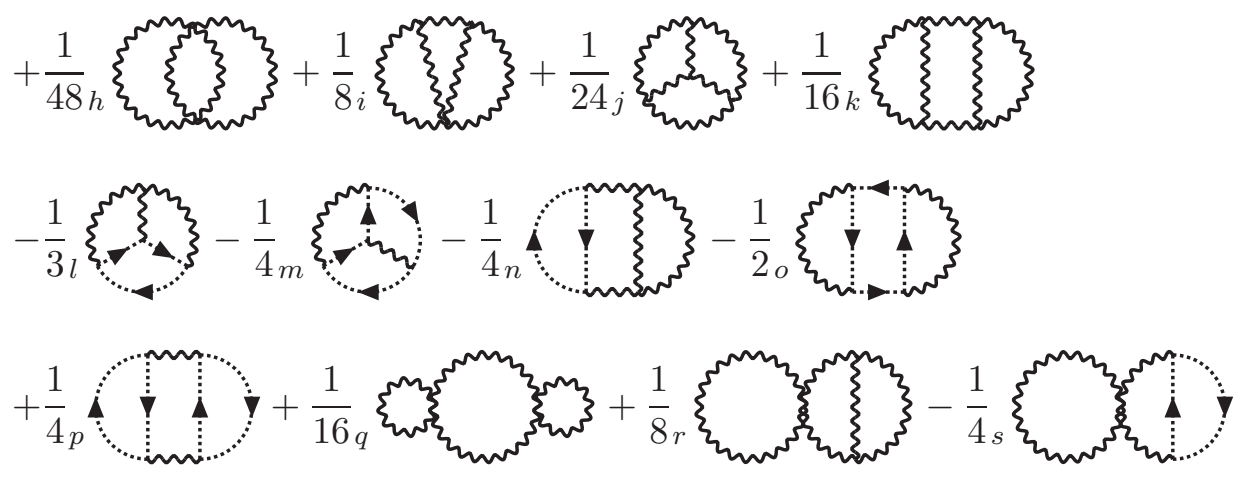

Figure 3. Three-loop diagrams contributing to $C_{\mathrm{A}}^{2}$.

e.g. in [19], all 2-loop sum-integrals factor into products of two 1-loop cases; this observation we reproduce via IBP, for all possible 2-loop vacuum sum-integrals, also of different dimensionality needed for other computations; see also Appendix A)

$$
\begin{aligned}
& p_{\mathrm{h}}^{[d]}=g^{2} d_{\mathrm{A}} C_{\mathrm{A}}\left(\frac{d(d-3)}{16} \xi^{2}+\frac{d}{2} \xi-\frac{d(d+1)}{4}\right) I_{1}^{0} I_{1}^{0}, \\
& p_{\mathrm{h}}^{[e]}=g^{2} d_{\mathrm{A}} C_{\mathrm{A}}\left(-\frac{d(d-3)}{16} \xi^{2}-\frac{4 d+1}{8} \xi+\frac{3 d}{4}\right) I_{1}^{0} I_{1}^{0}, \\
& p_{\mathrm{h}}^{[f]}=g^{2} d_{\mathrm{A}} C_{\mathrm{A}}\left(\frac{1}{8} \xi-\frac{1}{4}\right) I_{1}^{0} I_{1}^{0}, \\
& p_{\mathrm{h}}^{[g]}=g^{2} d_{\mathrm{A}} N_{\mathrm{f}} \frac{d-1}{2}\left(2 I_{1}^{0}-\hat{I}_{1}^{0}\right) \hat{I}_{1}^{0}=g^{2} d_{\mathrm{A}} N_{\mathrm{f}} \frac{d-1}{2}\left(2^{4-d}-4^{2-d}-3\right) I_{1}^{0} I_{1}^{0} .
\end{aligned}
$$

Turning now to the three-loop case, the IBP reduction leaves us with a set of bosonic

$$
\begin{aligned}
& B_{1} \equiv I_{112000 ; 000}^{000}=I_{1}^{0} I_{1}^{0} I_{2}^{0}, \quad B_{2} \equiv I_{110011 ; 000}^{000}, \\
& B_{3} \equiv I_{21111-2 ; 000}^{000} \quad B_{4} \equiv I_{110012 ; 000}^{200}, \quad B_{5} \equiv I_{11-2013 ; 000}^{000},
\end{aligned}
$$

as well as fermionic master sum-integrals of the form Eq. (3.1) (note however the linear relation Eq. (3.8) that can still be used to eliminate one of $B_{2}, F_{6}, F_{7}$ )

$$
\begin{array}{ll}
F_{1} \equiv I_{121000 ; 001}^{000}=I_{1}^{0} I_{2}^{0} \hat{I}_{1}^{0}=\left(2^{2-d}-1\right) B_{1}, & F_{6} \equiv I_{110011 ; 001}^{000} \\
F_{2} \equiv I_{112000 ; 001}^{000}=I_{1}^{0} I_{1}^{0} \hat{I}_{2}^{0}=\left(2^{4-d}-1\right) B_{1}, & F_{7} \equiv I_{1100011 ; 110}^{000} \\
F_{3} \equiv I_{1120000 ; 011}^{000}=I_{1}^{0} \hat{I}_{1}^{0} \hat{I}_{2}^{0}=\left(2^{2-d}-1\right)\left(2^{4-d}-1\right) B_{1}, & F_{8} \equiv I_{21111-2 ; 001}^{000} \\
F_{4} \equiv I_{2110000 ; 011}^{000}=I_{2}^{0} \hat{I}_{1}^{0} \hat{I}_{1}^{0}=\left(2^{2-d}-1\right)^{2} B_{1}, & F_{9} \equiv I_{21111-2 ; 011}^{000} \\
F_{5} \equiv I_{112000 ; 111}^{000}=\hat{I}_{1}^{0} \hat{I}_{1}^{0} \hat{I}_{2}^{0}=\left(2^{2-d}-1\right)^{2}\left(2^{4-d}-1\right) B_{1}, & F_{10} \equiv I_{11111-1 ; 100}^{000}
\end{array}
$$

In terms of these masters, we obtain $d$-dimensional bare results from the three-loop diagrams depicted in Fig. (3):

$$
p_{\mathrm{h}}^{[h+i+j+k]}=g^{4} d_{\mathrm{A}} C_{\mathrm{A}}^{2}\left(B _ { 1 } \left(\frac{d\left(2+33 d-12 d^{2}+d^{3}\right)}{384} \xi^{4}+\frac{d\left(-6-13 d+3 d^{2}\right)}{32} \xi^{3}+\right.\right.
$$




$$
\begin{aligned}
& \left.+\frac{2+41 d+56 d^{2}-8 d^{3}}{64} \xi^{2}-\frac{13+115 d+38 d^{2}-50 d^{3}+8 d^{4}}{8(d-3)(d-5)} \xi-\frac{3-14 d+4 d^{2}}{8}\right)+ \\
& +B_{2}\left(\frac{22-13 d+3 d^{2}}{768} \xi^{2}-\frac{11-19 d+6 d^{2}}{192(d-3)} \xi+\frac{5-10 d+4 d^{2}}{32}\right)+ \\
& \left.+B_{3} \frac{(2 d-1)^{2}}{16}-B_{4}\left(\frac{d-3}{32} \xi^{2}-\frac{1}{4} \xi\right)-B_{5}\left(\frac{1}{64} \xi^{2}-\frac{1}{8(d-3)} \xi\right)\right), \\
& p_{\mathrm{h}}^{[l]}=g^{4} d_{\mathrm{A}} C_{\mathrm{A}}^{2}\left(B_{1}\left(\frac{d-2}{16} \xi^{2}-\frac{d-2}{8(d-3)} \xi\right)-\right. \\
& -B_{2}\left(\frac{(d-1)(3 d-10)}{384} \xi^{2}+\frac{28-17 d+3 d^{2}}{96(d-3)} \xi+\frac{1}{16}\right)+ \\
& \left.+B_{4}\left(-\frac{d-3}{8} \xi^{2}+\frac{1}{4} \xi\right)+B_{5}\left(-\frac{1}{16} \xi^{2}+\frac{1}{8(d-3)} \xi\right)\right) \\
& p_{\mathrm{h}}^{[m]}=g^{4} d_{\mathrm{A}} C_{\mathrm{A}}^{2}\left(B_{1} \frac{d-2}{64} \xi^{2}+B_{2}\left(-\frac{(d-2)(3 d-7)}{768} \xi^{2}+\frac{5}{192} \xi-\frac{1}{32}\right)-\right. \\
& \left.-B_{4} \frac{d-3}{32} \xi^{2}-B_{5} \frac{1}{64} \xi^{2}\right) \\
& p_{\mathrm{h}}^{[n]}=g^{4} d_{\mathrm{A}} C_{\mathrm{A}}^{2}\left(B_{1}\left(-\frac{d(d-5)}{32} \xi^{3}+\frac{d(d-10)}{16} \xi^{2}+\frac{31+22 d-20 d^{2}+3 d^{3}}{4(d-3)(d-5)} \xi+\frac{2 d-3}{4}\right)+\right. \\
& +B_{2}\left(\frac{(3 d-1)(d-4)}{384} \xi^{2}+\frac{22-15 d+3 d^{2}}{48(d-3)} \xi+\frac{3+d}{16}\right)+ \\
& \left.+B_{3} \frac{1-2 d}{8}+B_{4}\left(\frac{d-3}{8} \xi^{2}-\frac{1}{2} \xi\right)+B_{5}\left(\frac{1}{16} \xi^{2}-\frac{1}{4(d-3)} \xi\right)\right), \\
& p_{\mathrm{h}}^{[o]}=g^{4} d_{\mathrm{A}} C_{\mathrm{A}}^{2}\left(B_{1} \frac{2-d}{32} \xi^{2}-B_{2}\left(\frac{1}{96} \xi^{2}-\frac{1}{24} \xi+\frac{1}{8}\right)+B_{4} \frac{d-3}{16} \xi^{2}+B_{5} \frac{1}{32} \xi^{2}\right), \\
& p_{\mathrm{h}}^{[p]}=g^{4} d_{\mathrm{A}} C_{\mathrm{A}}^{2}\left(B_{1}\left(\frac{1}{16} \xi^{2}-\frac{1}{8} \xi-\frac{1}{8}\right)+B_{2}\left(\frac{1}{64} \xi^{2}-\frac{1}{32} \xi\right)+B_{3} \frac{1}{16}\right), \\
& p_{\mathrm{h}}^{[q]}=g^{4} d_{\mathrm{A}} C_{\mathrm{A}}^{2} d\left(\frac{2+33 d-12 d^{2}+d^{3}}{384} \xi^{4}-\frac{1+14 d-3 d^{2}}{32} \xi^{3}+\right. \\
& \left.+\frac{1+15 d-2 d^{2}}{16} \xi^{2}-d \xi+\frac{d(d+1)}{4}\right) B_{1} \\
& p_{\mathrm{h}}^{[r]}=g^{4} d_{\mathrm{A}} C_{\mathrm{A}}^{2}\left(-\frac{d\left(2+33 d-12 d^{2}+d^{3}\right)}{192} \xi^{4}+\frac{d\left(7+27 d-6 d^{2}\right)}{32} \xi^{3}-\right. \\
& \left.-\frac{d\left(11+29 d-4 d^{2}\right)}{16} \xi^{2}+\frac{2-21 d-33 d^{2}+8 d^{3}}{4(d-5)} \xi-\frac{3 d^{2}}{2}\right) B_{1}, \\
& p_{\mathrm{h}}^{[s]}=g^{4} d_{\mathrm{A}} C_{\mathrm{A}}^{2}\left(\frac{d(d-5)}{32} \xi^{3}-\frac{d(d-9)}{16} \xi^{2}+\frac{2+13 d-3 d^{2}}{4(d-5)} \xi+\frac{d}{2}\right) B_{1} .
\end{aligned}
$$

The three-loop diagrams depicted in Fig. (4) can be expressed as (diagram $u \sim d_{\mathrm{A}} / N_{\mathrm{c}}=$ $d_{\mathrm{A}}\left(C_{\mathrm{A}}-2 C_{\mathrm{F}}\right)$ contributes to two color structures $)$

$$
p_{\mathrm{h}}^{[t+u]}=g^{4} d_{\mathrm{A}} N_{\mathrm{f}} C_{\mathrm{F}} \frac{1-d}{4}\left(2(d-1)\left(F_{2}-2 F_{3}+F_{5}+F_{10}\right)+4 F_{6}+(d-5) F_{7}\right),
$$




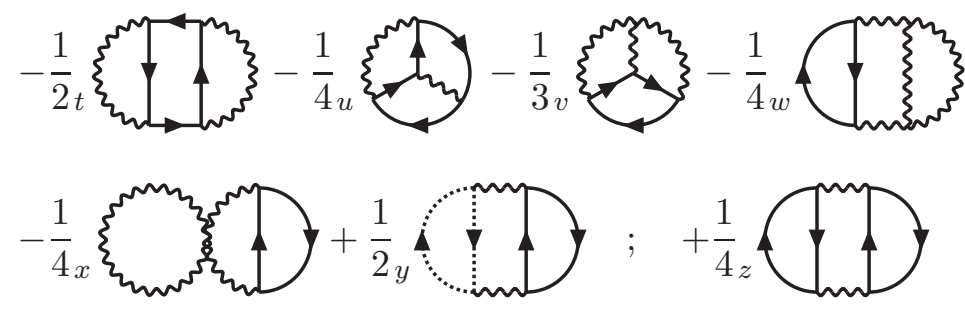

Figure 4. Three-loop diagrams contributing to $N_{\mathrm{f}}$, and to $N_{\mathrm{f}}^{2}$ (last diagram only).

$$
\begin{aligned}
p_{\mathrm{h}}^{[u+v+w]} & =g^{4} d_{\mathrm{A}} N_{\mathrm{f}} C_{\mathrm{A}}\left(\left(\frac{2(d-1)}{d-5} \xi+(5 d-3)\right) F_{1}-\frac{2-4 d+d^{2}}{4} F_{6}+\right. \\
& \left.+\frac{(d-1)(d-5)}{8} F_{7}-\frac{2 d-1}{2} F_{8}\right) \\
p_{\mathrm{h}}^{[x]} & =g^{4} d_{\mathrm{A}} N_{\mathrm{f}} C_{\mathrm{A}}(1-d)\left(\frac{2}{d-5} \xi+d\right) F_{1} \\
p_{\mathrm{h}}^{[y]} & =g^{4} d_{\mathrm{A}} N_{\mathrm{f}} C_{\mathrm{A}}\left(-2 F_{1}-\frac{1}{4} F_{6}+\frac{1}{2} F_{8}\right)
\end{aligned}
$$

The last three-loop diagram depicted in Fig. (4) gives

$$
p_{\mathrm{h}}^{[z]}=g^{4} d_{\mathrm{A}} N_{\mathrm{f}}^{2}\left((d-5) F_{4}+\frac{d-3}{4} F_{7}+F_{9}\right) .
$$

Summing all diagrams, the gauge parameter $\xi$ explicitly drops out (and so do the 3-loop sum-integrals $B_{4}$ and $B_{5}$ ), leaving the (bare) result

$$
\begin{aligned}
p_{\mathrm{h}}(T) & =\frac{1}{d}\left((1-d) d_{\mathrm{A}}+4\left(2^{-d}-1\right) N_{\mathrm{f}} C_{\mathrm{A}}\right) I_{1}^{2}+ \\
& +g^{2} d_{\mathrm{A}} \frac{1-d}{4}\left((d-1) C_{\mathrm{A}}+2\left(3+4^{2-d}-2^{4-d}\right) N_{\mathrm{f}}\right) I_{1}^{0} I_{1}^{0}+ \\
& +g^{4} d_{\mathrm{A}} C_{\mathrm{A}}^{2} \frac{(1-d)^{2}}{8}\left(2(d-5) B_{1}+B_{2}+2 B_{3}\right)+ \\
& +g^{4} d_{\mathrm{A}} N_{\mathrm{f}} C_{\mathrm{A}} \frac{1-d}{8}\left(8(d-5) F_{1}+2(d-3) F_{6}-(d-5) F_{7}+8 F_{8}\right)+ \\
& +g^{4} d_{\mathrm{A}} N_{\mathrm{f}} C_{\mathrm{F}} \frac{1-d}{4}\left(2(d-1)\left(F_{2}-2 F_{3}+F_{5}+F_{10}\right)+4 F_{6}+(d-5) F_{7}\right)+ \\
& +g^{4} d_{\mathrm{A}} N_{\mathrm{f}}^{2} \frac{1}{4}\left(4(d-5) F_{4}+(d-3) F_{7}+4 F_{9}\right)+\mathcal{O}\left(g^{6}\right) .
\end{aligned}
$$

Note that also the spurious poles (at $d=3-2 \epsilon$ ) which are present in Eqs. (4.18), (4.19) and (4.21) (and which would have severely limited our ability to evaluate the constant term, requiring 3-loop sum-integrals to higher order in $\epsilon$ ) have canceled completely. Comparing with the corresponding expression given in Eq. (31) of [19], we note complete agreement, up 
to two typos in that reference (in their 3-loop terms, $C_{\mathrm{A}} T_{\mathrm{F}} \mathcal{I}_{1} \mathcal{I}_{2} \tilde{\mathcal{I}}_{1}$ is missing a prefactor $(1+\epsilon)$, and $C_{\mathrm{F}} T_{\mathrm{F}} \mathcal{I}_{1} \tilde{\mathcal{I}}_{1} \tilde{\mathcal{I}}_{2}$ should be multiplied by $1 / 2$; these correspond to our structures $N_{\mathrm{f}} C_{\mathrm{A}} F_{1}$ and $N_{\mathrm{f}} C_{\mathrm{F}} F_{3}$ above).

Renormalizing the coupling in the $\overline{\mathrm{MS}}$ scheme via

$$
g^{2}=\mu^{-2 \epsilon} g_{\text {bare }}^{2}=g_{\mathrm{R}}^{2}\left(1-\frac{\beta_{0}}{\epsilon} \frac{g_{\mathrm{R}}^{2}}{16 \pi^{2}}+\mathcal{O}\left(g_{\mathrm{R}}^{4}\right)\right), \quad \beta_{0}=\frac{11 C_{\mathrm{A}}-2 N_{\mathrm{f}}}{3}
$$

from which (using that $g_{\text {bare }}^{2}$ does not depend on the renormalization scale $\mu$ ) its running

$g_{\mathrm{R}}^{2}(\mu)=g_{\mathrm{R}}^{2}\left(\mu_{0}\right)\left(1+2 \beta_{0} \frac{g_{\mathrm{R}}^{2}}{16 \pi^{2}} \ln \frac{\mu_{0}}{\mu}+\mathcal{O}\left(g_{\mathrm{R}}^{4}\right)\right)$ follows, and expanding the master sum-integrals around $d=3-2 \epsilon$ (see Appendix), the result coincides with the expression given in Eq. (32) of [19] (note that they write the $\overline{\mathrm{MS}}$ scale parameter as $\Lambda$, where $\Lambda^{2} \equiv \bar{\mu}^{2} \equiv 4 \pi e^{-\gamma_{\mathrm{E}}} \mu^{2}$ ), which has subsequently been used to build $p_{\mathrm{QCD}}(T)$ according to Eq. (2.3). We will not re-iterate discussions about convergence as well as (renormalization) scale dependence here, but instead only refer to the literature [18, 32-34]. Our main point was the re-derivation of $p_{\text {hard }}(T)$ in Eq. (4.32), demonstrating gauge-parameter independence and using automated computer-algebra methods, which are naturally extensible to higher orders.

\section{Conclusions}

We have re-derived the results of the original 3-loop computation [19] for the QCD pressure. While the original calculation was performed in Feynman gauge, we have used covariant gauge and shown explicit cancellation of the gauge parameter. As a slight improvement over [19], our IBP reduction revealed that the basis of non-trivial 3-loop master integrals used in the original computation could be reduced by one.

In future work, we hope to be able to employ the IBP setup to the 4-loop level, thus contributing to the last missing piece needed for the physical leading-order (i.e. $g^{6}$ ) determination of the pressure within the dimensionally reduced effective theory framework $[24,26,32]$ - the only currently known framework allowing for a weak-coupling expansion of thermodynamic observables that is systematically improvable.

\section{Acknowledgments}

We are indebted to Shin-ichiro Hara for advice on his algebraic library for Ruby. M.N. thanks the theory group of Bielefeld University for hospitality during the period when this work was done. The work of Y.S. is supported by the Heisenberg Programme of the Deutsche Forschungsgemeinschaft (DFG grant SCHR 993/1-1). All figures were drawn with Axodraw [35].

\section{A One- and two-loop vacuum sum-integrals}

The one-loop bosonic tadpole is known analytically and reads $(d=3-2 \epsilon)$

$$
I_{m}^{n} \equiv \oiint_{P} \frac{P_{0}^{n}}{\left(P^{2}\right)^{m}}=\frac{2 \pi^{3 / 2} T^{4}}{(2 \pi T)^{2 m-n}}\left(\frac{\mu^{2}}{\pi T^{2}}\right)^{\epsilon} \frac{\Gamma\left(m-\frac{3}{2}+\epsilon\right)}{\Gamma(m)} \zeta(2 m-n-3+2 \epsilon),
$$


whereas the fermionic tadpole can be related to the corresponding bosonic one via

$$
\hat{I}_{m}^{n} \equiv \sum_{\{P\}} \frac{P_{0}^{n}}{\left(P^{2}\right)^{m}}=\left(2^{2 m-n-d}-1\right) I_{m}^{n},
$$

which immediately follows from the scaling relations explained in Sec. 3.

As mentioned above, via integration-by-parts relations all two-loop integrals are expressible in terms of products of two one-loop tadpoles which means they are also available analytically up to arbitrary order in $\epsilon$. A typical reduction, following from IBP and being used in the 2-loop contribution to $p_{\text {hard }}(T)$, reads

$$
\mathcal{S} \equiv \sum_{P Q} \frac{1}{P^{2} Q^{2}(P-Q)^{2}}=0 .
$$

This remarkable result in fact follows from the IBP relation

$$
\begin{aligned}
& 0=\sum_{P Q} \partial_{p_{i}} f_{i} \frac{1}{P^{2} Q^{2}(P-Q)^{2}} \\
& \text { with } f_{i}=(d-2)\left(p_{i}+q_{i}\right)+\frac{2}{Q^{2}}\left(P_{0}+Q_{0}\right)\left(q_{i} P_{0}-p_{i} Q_{0}\right),
\end{aligned}
$$

from which, after working out the derivatives, using the shift $Q \rightarrow P-Q$ and exploiting the $P \leftrightarrow Q$ symmetry, it follows that $0=(d-2)(d-3) \mathcal{S}$, proving Eq. (A.3). Note that in the literature, by explicit integration Eq. (A.3) was only known to hold to $\mathcal{O}(\epsilon)[18]$.

\section{B Three-loop vacuum sum-integrals}

All non-trivial three-loop master sum-integrals that are needed for $p_{\text {hard }}$ of Eq. (4.32) have been evaluated in Ref. [18], and been subsequently summarized in the literature [19, 22]. In the notation of Sec. 4, they read

$$
\begin{aligned}
& B_{2}=\frac{T^{4}}{16 \pi^{2}}\left(\frac{\mu^{2}}{4 \pi T^{2}}\right)^{3 \epsilon} \frac{1}{\epsilon} \frac{1}{24}\left[1+\left(\frac{91}{15}-3 \gamma_{\mathrm{E}}+8 Z_{1}-2 Z_{3}\right) \epsilon+\mathcal{O}(\epsilon)^{2}\right], \\
& B_{3}=\frac{T^{4}}{16 \pi^{2}}\left(\frac{\mu^{2}}{4 \pi T^{2}}\right)^{3 \epsilon} \frac{1}{\epsilon} \frac{11}{216}\left[1+\left(\frac{73}{22}-\frac{21}{11} \gamma_{\mathrm{E}}+\frac{64}{11} Z_{1}-\frac{10}{11} Z_{3}\right) \epsilon+\mathcal{O}(\epsilon)^{2}\right], \\
& F_{7}=\frac{T^{4}}{16 \pi^{2}}\left(\frac{\mu^{2}}{4 \pi T^{2}}\right)^{3 \epsilon} \frac{1}{\epsilon} \frac{1}{96}\left[1+\left(\frac{173}{30}-3 \gamma_{\mathrm{E}}-\frac{42}{5} \ln 2+8 Z_{1}-2 Z_{3}\right) \epsilon+\mathcal{O}(\epsilon)^{2}\right], \\
& F_{8}=\frac{T^{4}}{16 \pi^{2}}\left(\frac{\mu^{2}}{4 \pi T^{2}}\right)^{3 \epsilon} \frac{1}{\epsilon} \frac{-29}{1728}\left[1+\left(\frac{89}{29}-\frac{39}{29} \gamma_{\mathrm{E}}-\frac{90}{29} \ln 2+\frac{136}{29} Z_{1}-\frac{10}{29} Z_{3}\right) \epsilon+\mathcal{O}(\epsilon)^{2}\right], \\
& F_{9}=\frac{T^{4}}{16 \pi^{2}}\left(\frac{\mu^{2}}{4 \pi T^{2}}\right)^{3 \epsilon} \frac{1}{\epsilon} \frac{1}{108}\left[1+\left(\frac{35}{8}-\frac{3}{2} \gamma_{\mathrm{E}}-\frac{63}{10} \ln 2+5 Z_{1}-\frac{1}{2} Z_{3}\right) \epsilon+\mathcal{O}(\epsilon)^{2}\right], \\
& F_{10}=\frac{T^{4}}{16 \pi^{2}}\left(\frac{\mu^{2}}{4 \pi T^{2}}\right)^{3 \epsilon} \frac{1}{\epsilon} \frac{-1}{192}\left[1+\left(\frac{361}{60}+3 \gamma_{\mathrm{E}}+\frac{76}{5} \ln 2-4 Z_{1}+4 Z_{3}\right) \epsilon+\mathcal{O}(\epsilon)^{2}\right],
\end{aligned}
$$

where $Z_{i} \equiv \frac{\zeta^{\prime}(-i)}{\zeta(-i)}$. $F_{6}$ can be obtained via Eq. (3.8), and coincides with the expansion given in $[19]$. 


\section{References}

[1] P. A. Baikov, K. G. Chetyrkin, J. H. Kühn and J. Rittinger, Vector Correlator in Massless $Q C D$ at Order $O\left(\alpha_{s}^{4}\right)$ and the QED beta-function at five loop, JHEP 1207 (2012) 017 [arXiv:1206.1284 [hep-ph]].

[2] S. Laporta and E. Remiddi, The Analytical value of the electron (g-2) at order alpha**3 in QED, Phys. Lett. B 379 (1996) 283 [hep-ph/9602417]; S. Laporta and E. Remiddi, Status of the QED prediction of the electron (g - 2), Nucl. Phys. Proc. Suppl. 181-182 (2008) 10.

[3] Y. Schröder and M. Steinhauser, Four-loop singlet contribution to the rho parameter, Phys. Lett. B 622 (2005) 124 [hep-ph/0504055]; R. Boughezal and M. Czakon, Single scale tadpoles and $O\left(G\left(F m(t) * *_{2}\right.\right.$ alpha $\left.\left.(s)^{* * 3}\right)\right)$ corrections to the rho parameter, Nucl. Phys. B 755 (2006) 221 [hep-ph/0606232].

[4] P. Nogueira, Automatic Feynman graph generation, J. Comput. Phys. 105 (1993) 279; P. Nogueira, Abusing qgraf, Nucl. Instrum. Meth. A 559 (2006) 220.

[5] J. A. M. Vermaseren, New features of FORM, math-ph/0010025; J. Kuipers, T. Ueda, J. A. M. Vermaseren and J. Vollinga, FORM version 4.0, arXiv:1203.6543 [cs.SC].

[6] C. W. Bauer, A. Frink and R. Kreckel, Introduction to the GiNaC framework for symbolic computation within the $\mathrm{C}++$ programming language, cs/0004015 [cs-sc].

[7] Wolfram Research, Inc., Mathematica, Version 8.0, Champaign, IL (2012).

[8] K. G. Chetyrkin and F. V. Tkachov, Integration by Parts: The Algorithm to Calculate beta Functions in 4 Loops, Nucl. Phys. B 192 (1981) 159; F. V. Tkachov, A Theorem on Analytical Calculability of Four Loop Renormalization Group Functions, Phys. Lett. B 100 (1981) 65.

[9] S. Laporta, High precision calculation of multiloop Feynman integrals by difference equations, Int. J. Mod. Phys. A 15 (2000) 5087 [hep-ph/0102033].

[10] C. Anastasiou and A. Lazopoulos, Automatic integral reduction for higher order perturbative calculations, JHEP 0407 (2004) 046 [hep-ph/0404258].

[11] A. V. Smirnov, Algorithm FIRE - Feynman Integral REduction, JHEP 0810 (2008) 107 [arXiv:0807.3243 [hep-ph]].

[12] C. Studerus, Reduze-Feynman Integral Reduction in $\mathrm{C}++$, Comput. Phys. Commun. 181 (2010) 1293 [arXiv:0912.2546 [physics.comp-ph]]; A. von Manteuffel and C. Studerus, Reduze 2 - Distributed Feynman Integral Reduction, arXiv:1201.4330 [hep-ph].

[13] S. Laporta, P. Mastrolia and E. Remiddi, The Analytic value of a four loop sunrise graph in a particular kinematical configuration, Nucl. Phys. B 688 (2004) 165 [hep-ph/0311255]; M. Argeri and P. Mastrolia, Feynman Diagrams and Differential Equations, Int. J. Mod. Phys. A 22 (2007) 4375 [arXiv:0707.4037 [hep-ph]].

[14] E. Remiddi and J. A. M. Vermaseren, Harmonic polylogarithms, Int. J. Mod. Phys. A 15 (2000) 725 [hep-ph/9905237]; J. A. M. Vermaseren, Harmonic sums, Mellin transforms and integrals, Int. J. Mod. Phys. A 14 (1999) 2037 [hep-ph/9806280].

[15] S. Moch, P. Uwer and S. Weinzierl, Nested sums, expansion of transcendental functions and multiscale multiloop integrals, J. Math. Phys. 43 (2002) 3363 [hep-ph/0110083]. 
[16] C. Bogner and S. Weinzierl, Feynman graph polynomials, Int. J. Mod. Phys. A 25 (2010) 2585 [arXiv:1002.3458 [hep-ph]].

[17] G. Heinrich, Sector Decomposition, Int. J. Mod. Phys. A 23 (2008) 1457 [arXiv:0803.4177 [hep-ph]]; S. Borowka, J. Carter and G. Heinrich, Numerical evaluation of multi-loop integrals for arbitrary kinematics with SecDec 2.0, arXiv:1204.4152 [hep-ph].

[18] P. B. Arnold and C. X. Zhai, The Three loop free energy for pure gauge QCD, Phys. Rev. D 50 (1994) 7603 [hep-ph/9408276]; P. B. Arnold and C. X. Zhai, The Three loop free energy for high temperature QED and QCD with fermions, Phys. Rev. D 51 (1995) 1906 [hep-ph/9410360].

[19] E. Braaten and A. Nieto, Free energy of QCD at high temperature, Phys. Rev. D 53 (1996) 3421 [hep-ph/9510408].

[20] J. O. Andersen, L. E. Leganger, M. Strickland and N. Su, Three-loop HTL QCD thermodynamics, JHEP 1108 (2011) 053 [arXiv:1103.2528 [hep-ph]]; J. Möller and Y. Schröder, Three-loop matching coefficients for hot QCD: Reduction and gauge independence, arXiv:1207.1309 [hep-ph].

[21] A. Gynther, M. Laine, Y. Schröder, C. Torrero and A. Vuorinen, Four-loop pressure of massless O(N) scalar field theory, JHEP 0704 (2007) 094 [hep-ph/0703307 [HEP-PH]]; J. O. Andersen and L. Kyllingstad, Four-loop Screened Perturbation Theory, Phys. Rev. D 78 (2008) 076008 [arXiv:0805.4478 [hep-ph]]; A. Gynther, A. Kurkela and A. Vuorinen, The $N(f)^{* *} 3 g^{* *} 6$ term in the pressure of hot QCD, Phys. Rev. D 80 (2009) 096002 [arXiv:0909.3521 [hep-ph]].

[22] Y. Schröder, Loops for Hot QCD, Nucl. Phys. Proc. Suppl. 183B (2008) 296 [arXiv:0807.0500 [hep-ph]].

[23] J. Möller and Y. Schröder, Three-loop matching coefficients for hot QCD: Reduction and gauge independence, arXiv:1207.1309 [hep-ph]; J. Möller and Y. Schröder, Open problems in hot QCD, Nucl. Phys. Proc. Suppl. 205-206 (2010) 218 [arXiv:1007.1223 [hep-ph]].

[24] E. Braaten and A. Nieto, Effective field theory approach to high temperature thermodynamics, Phys. Rev. D 51 (1995) 6990 [hep-ph/9501375].

[25] P. H. Ginsparg, First Order and Second Order Phase Transitions in Gauge Theories at Finite Temperature, Nucl. Phys. B 170 (1980) 388; T. Appelquist and R. D. Pisarski,

High-Temperature Yang-Mills Theories and Three-Dimensional Quantum Chromodynamics, Phys. Rev. D 23 (1981) 2305; K. Kajantie, M. Laine, K. Rummukainen and M. E. Shaposhnikov, Generic rules for high temperature dimensional reduction and their application to the standard model, Nucl. Phys. B 458 (1996) 90 [hep-ph/9508379].

[26] A. Hietanen, K. Kajantie, M. Laine, K. Rummukainen and Y. Schröder, Plaquette expectation value and gluon condensate in three dimensions, JHEP 0501 (2005) 013 [hep-lat/0412008];

F. Di Renzo, M. Laine, V. Miccio, Y. Schröder and C. Torrero, The Leading non-perturbative coefficient in the weak-coupling expansion of hot QCD pressure, JHEP 0607 (2006) 026 [hep-ph/0605042].

[27] Y. Schröder, Weak-coupling expansion of the hot QCD pressure, PoS JHW 2005 (2006) 029 [hep-ph/0605057].

[28] A. D. Linde, Infrared Problem in Thermodynamics of the Yang-Mills Gas, Phys. Lett. B 96 (1980) 289. 
[29] Ruby, http://www.ruby-lang.org.

[30] P. B. Arnold and O. Espinosa, The Effective potential and first order phase transitions: Beyond leading-order, Phys. Rev. D 47 (1993) 3546 [Erratum-ibid. D 50 (1994) 6662] [hep-ph/9212235].

[31] C. Coriano and R. R. Parwani, The Three loop equation of state of QED at high temperature, Phys. Rev. Lett. 73 (1994) 2398 [hep-ph/9405343]; R. R. Parwani and C. Coriano, Higher order corrections to the equation of state of QED at high temperature, Nucl. Phys. B 434 (1995) 56 [hep-ph/9409269].

[32] K. Kajantie, M. Laine, K. Rummukainen and Y. Schröder, How to resum long distance contributions to the QCD pressure?, Phys. Rev. Lett. 86 (2001) 10 [hep-ph/0007109]; K. Kajantie, M. Laine, K. Rummukainen and Y. Schröder, The Pressure of hot QCD up to g6 $\ln (1 / g)$, Phys. Rev. D 67 (2003) 105008 [hep-ph/0211321].

[33] E. Braaten and A. Nieto, On the convergence of perturbative QCD at high temperature, Phys. Rev. Lett. 76 (1996) 1417 [hep-ph/9508406].

[34] A. Hietanen, K. Kajantie, M. Laine, K. Rummukainen and Y. Schröder, Three-dimensional physics and the pressure of hot QCD, Phys. Rev. D 79 (2009) 045018 [arXiv:0811.4664 [hep-lat]].

[35] J. A. M. Vermaseren, Axodraw, Comput. Phys. Commun. 83 (1994) 45. 